01

\title{
Энергия связи основного состояния бериллиеподобного молибдена: корреляционные и квантово-электродинамические эффекты
}

\author{
(C) А.В. Малышев, Ю.С. Кожедуб, И.С. Анисимова, Д.А. Глазов, М.Ю. Кайгородов, \\ И.И. Тупицын, В.М. Шабаев \\ Санкт-Петербургский государственный университет, \\ 199034 Санкт-Петербург, Россия \\ e-mail: a.v.malyshev@spbu.ru
}

Поступила в редакцию 15.01.2021 г.

В окончательной редакции 15.01.2021 г.

Принята к публикации 20.01.2021 г.

Выполнены высокоточные расчеты энергии связи основного состояния бериллиеподобного молибдена $(Z=42)$. Примененный подход совмещает строгое квантово-электродинамическое (КЭД) рассмотрение вплоть до второго порядка теории возмущений в рамках картины Фарри и учет корреляционных эффектов третьего и более высоких порядков в брейтовском приближении. Примешивание близких конфигураций одинаковой симметрии за счет эффектов электрон-электронного взаимодействия рассмотрено, использую КЭД теорию возмущений для квазивырожденных уровней. Получены наиболее точные на сегодняшний день теоретические предсказания для энергии связи.

Ключевые слова: релятивистская теория атома, квантовая электродинамика, корреляционные эффекты, многозарядные ионы, теория возмущений для квазивырожденных уровней.

DOI: $10.21883 /$ OS.2021.05.50882.1814-21

\section{1. Введение}

Среди наиболее интересных и перспективных систем для тестирования теоретических методов квантовой электродинамики особое место занимают системы, в которых электроны подвержены действию сильных электромагнитных полей. Ярким примером подобных систем служат многозарядные ионы [1-3]. В отличие от легких атомов в многозарядных ионах параметр $\alpha Z$ ( $\alpha$ - постоянная тонкой структуры, $Z-$ заряд ядра) не является малым. Как следствие, оказываются неприменимыми методы, учитывающие взаимодействие с полем ядра по теории возмущений $[4,5]$. Для описания этого режима сильной связи необходимо разрабатывать новые непертурбативные подходы. С другой стороны, относительно небольшое количество электронов в многозарядных ионах позволяет достаточно точно учитывать корреляционные эффекты, развивая теорию возмушений по параметру $1 / Z$. При этом теоретическая погрешность, связанная с расчетом данных вкладов, не маскирует представляющие наибольший интерес квантово-электродинамические (КЭД) эффекты, как это имеет место, например, для тяжелых нейтральных атомов.

В настоящее время наиболее строгая проверка КЭД методов расчета уровней энергии в многозарядных ионах связана с измерением лэмбовского сдвига в водородо- и литиеподобном уране [6-10]. По сравнению с наиболее простым случаем водородоподобных ионов $[11,12]$ теоретическое исследование литиеподобных систем осложняется не только необходимостью учета электрон-электронных корреляций [13-15], но и добавлением существенно новых многоэлектронных КЭД эффектов [16-18]. Однако в силу относительной простоты электронной конфигурации $1 s^{2} 2 l(l-$ орбитальное квантовое число валентного электрона) основного и ближайших возбужденных состояний литиеподобных ионов обе эти проблемы могут быть с успехом разрешены в рамках последовательной теории возмущений для одиночных (изолированных) уровней [19-21]. Мы не обсуждаем здесь возбуждения электронов из оболочки $1 s^{2}$, поскольку они приводят к формированию автоионизационных состояний [22,23], что выходит за рамки настоящего исследования. Квантовоэлектродинамические расчеты для конфигурации $1 s^{2}$, соответствующей основному состоянию гелиеподобных ионов, также не составляют проблемы [24]. С несколько более сложной конфигурацией $1 s 2 l$ с двумя открытыми оболочками приходится столкнуться, исследуя возбужденные состояния гелиеподобных ионов. При этом в отдельных случаях возникает необходимость прибегнуть к теории возмущений для квазивырожденных уровней. В последнее время значительный прогресс был достигнут в изучении гелиеподобных ионов как со стороны эксперимента [25-31], так и со стороны теории [32-37]. Следующим шагом является переход к рассмотрению бериллиеподобных ионов. Квантово-электродинамические эффекты здесь имеют тот же порядок величины, что и в гелие- и литиеподобных системах. С другой стороны, значительно возрастает роль корреляционных эффектов. Оказываются одинаково важными как взаимодействие электронов внутри оболочек, так и взаимодействие меж- 
ду различными оболочками. Сильное межэлектронное взаимодействие заметно перемешивает близкие уровни одинаковой симметрии. Так, например, на энергию связи основного состояния большое влияние оказывает примешивание конфигурации $1 s^{2} 2 p^{2}$ [38]. В результате высокоточные расчеты уровней энергии многозарядных бериллиеподобных ионов в рамках квантовой электродинамики для связанных состояний представляют собой весьма нетривиальную задачу. В литературе существует большое количество релятивистских расчетов бериллиеподобных ионов [39-48], но КЭД эффекты в них были рассмотрены в лучшем случае на основе некоторых одноэлектронных приближений. Вместе с тем прецизионные теоретические предсказания уровней энергии для бериллиеподобных ионов востребованы экспериментом [10,49-59]. Дополнительная мотивация обратиться к теоретическому исследованию четырехэлектронных систем заключается в том, что в работе [10] был измерен также переход $2 s 2{ }^{1} S_{0}-2 s 2 p^{3} P_{1}$ в бериллиеподобном уране (здесь и далее мы для краткости опускаем замкнутую $K$-оболочку в обозначениях уровней). При этом полученная точность даже превысила соответствующую точность, достигнутую для литиеподобного урана.

Данное исследование посвящено тщательному и всестороннему анализу энергии связи основного состояния бериллиеподобных ионов в рамках строгого полностью релятивистского подхода с учетом всех необходимых КЭД вкладов до второго порядка теории возмущений включительно. В работах $[60,61]$ нами были выполнены расчеты энергии связи с использованием КЭД теории возмущений для одиночного уровня (по аналогии с тем, как это было сделано для литиеподобных ионов [62]). Однако в силу упомянутой выше близости всех $n=2$ состояний реальная точность полученных теоретических предсказаний остается неясной до тех пор пока не будут осуществлены соответствующие КЭД расчеты с применением теории возмущений для квазивырожденных уровней. На решение данной проблемы и нацелена эта работа.

Более конкретно, в качестве объекта исследования нами был выбран ион молибдена $\mathrm{Mo}^{38+}$ в основном состоянии. Данный выбор объясняется несколькими причинами. Во-первых, этой работой мы хотим продемонстрировать принципиальную взаимосвязь корреляционных эффектов и КЭД эффектов (как одноэлектронных, так и многоэлектронных) в бериллиеподобных ионах. Квантово-электродинамические вклады быстро растут с увеличением заряда ядра $Z$, а роль электрон-электронного взаимодействия и смешивания уровней напротив ослабевает. В связи с этим оптимальной является область средних $Z$. Во-вторых, в области больших $Z$ и для урана, в частности, теоретическая погрешность энергии связи во многом определяется ядерной физикой, а именно неопределенностью в модели и размере атомного ядра и точностью, с которой оказывается возможным оценить поляризацию ядра. Эти эффекты лежат вне рамок квантовой электродинамики, поэтому мы предполагаем вернуться к обсуждению энергий связи для тяжелых бериллиеподобных ионов в наших последующих исследованиях. Наконец, расчеты энергии связи основного состояния являются важнейшим шагом на пути определения энергий переходов. Имея в виду прецизионные результаты измерений для переходов $2 s 2{ }^{1} S_{0}-2 s 2 p{ }^{1} P_{1}$ и $2 s 2{ }^{1} S_{0}-2 s 2 p^{3} P_{1}$ в бериллиеподобном молибдене [63], мы остановили свой выбор именно на этом элементе изоэлектронного ряда.

В статье использована релятивистская система единиц $(\hbar=1, c=1)$ и единицы Хэвисайда для заряда $\left(e^{2}=4 \pi \alpha, e<0\right)$.

\section{2. Теоретические методы}

Как было отмечено выше, для описания многозарядных ионов необходимо использовать формализм, в котором взаимодействие с ядром учтено во всех порядках по $\alpha Z$. Поэтому в качестве отправной точки для построения КЭД теории возмущений естественно использовать одночастичное уравнение Дирака

$$
\left[-i \boldsymbol{\alpha} \cdot \nabla+\beta m+V_{\text {nucl }}\right] \psi_{n}=\varepsilon_{n} \psi_{n},
$$

что соответствует квантовой электродинамике в картине Фарри [64]. Невозмущенные многоэлектронные волновые функции при этом могут быть построены в $j j$-связи из детерминантов Слейтера, составленных из решений уравнения (1). В качестве альтернативы уравнение (1) может быть модифицировано путем добавления к потенциалу ядра некоторого локального экранирующего потенциала, частично моделирующего эффекты межэлектронного взаимодействия: $V_{\text {nucl }} \rightarrow V_{\text {nucl }}+V_{\text {scr. }}$ Это соответствует расширенной версии картины Фарри [15,20,62,65-69]. Оставшаяся часть электрон-электронного взаимодействия, а также эффекты взаимодействия с квантованным электромагнитным полем должны быть учтены в рамках подходящей теории возмущений по $1 / Z$ и $\alpha$. Несмотря на то, что малый параметр $1 / Z$, строго говоря, соответствует только кулоновскому потенциалу и нерелятивистской теории, в данной работе мы используем его для обозначения порядка теории возмущений по остаточному межэлектронному взаимодействию.

Квантово-электродинамическая теория возмущений может быть сформулирована в рамках метода двухвременной функции Грина (TTGF) [70]. Для набора из $s$ квазивырожденных уровней, образующих модельное подпространство $\Omega$, метод TTGF предполагает построение $s \times s$ матрицы эффективного гамильтониана $H$. При этом энергии квазивырожденных состояний могут быть получены посредством диагонализации данной матрицы. Отметим, что стандартная невырожденная теория возмущений для одиночного уровня формально соответствует случаю $s=1$. В данной работе мы сравниваем три 


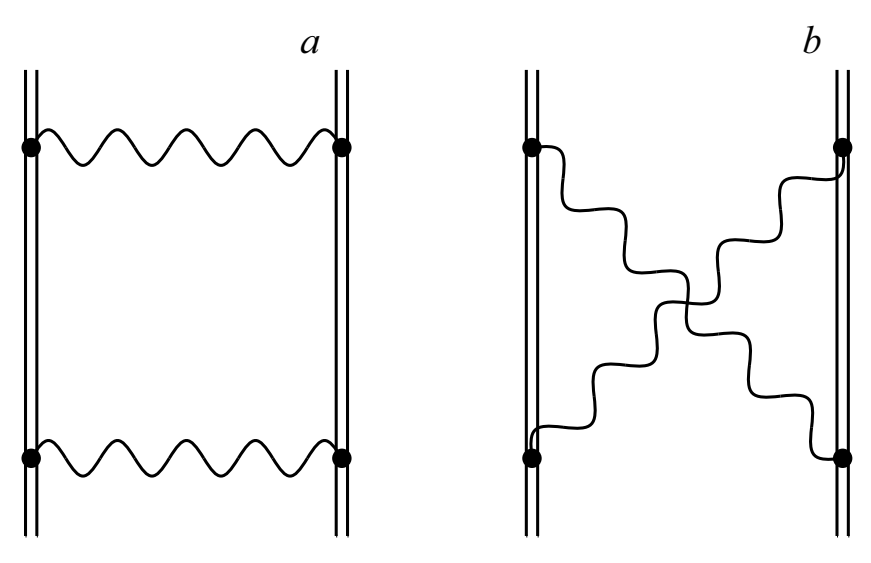

Диаграммы двухфотонного обмена.

альтернативные схемы для расчета энергии связи основного состояния бериллиеподобного молибдена. Более конкретно, исследуемое основное состояние $2 s 2 s{ }^{1} S_{0}$ рассматривается как: $(a)$ одиночный уровень $(2 s 2 s)_{0}$; (b) один уровень из пары квазивырожденных состояний, $(2 s 2 s)_{0}$ и $\left(2 p_{1 / 2} 2 p_{1 / 2}\right)_{0}$, с двумерным подпространством $\Omega ;(c)$ один уровень из тройки квазивырожденных состояний (с добавлением конфигурации $\left.\left(2 p_{3 / 2} 2 p_{3 / 2}\right)_{0}\right)$ с трехмерным подпространством $\Omega$. Схема $(a)$ воспроизводит подход, использованный нами ранее в работе [60]. В кулоновском потенциале энергии состояний $2 s, 2 p_{1 / 2}$ и $2 p_{3 / 2}$, которые определяют выбранные модельные подпространства, расщеплены только за счет конечного размера ядра и релятивистских эффектов. Отметим, что в общем случае применение расширенной версии картины Фарри сводится к перестройке рядов теории возмущений. Иногда данная процедура позволяет снять вырождение в нулевом приближении, так что необходимость в применении теории возмущений для квазивырожденных уровней может отпасть. Однако a priori это не известно.

Обсудим кратко используемый нами подход. Насколько нам известно, выполняемые здесь КЭД расчеты, которые охватывают все релевантные вклады вплоть до второго порядка теории возмущений для квазивырожденных уровней, никогда не предпринимались ранее для систем с более чем двумя электронами. Более того, соответствующие формальные выражения для двухэлектронных вкладов второго порядка были выведены методом TTGF только для случая двумерного модельного подпространства $\Omega$ [35-37,71,72]. Таким образом, во-первых, необходимо распространить формализм работы [37], примененный нами недавно для описания гелиеподобных ионов, на бериллиеподобные системы, и, во-вторых, обобщить его на случай модельного подпространства $\Omega$ произвольного размеpa $s$. Первую задачу можно решить, изменив знак перед $i 0$ в знаменателе электронного пропагатора, соответствующем $1 s$, и тем самым переопределив вакуумное состояние [69], отнеся к нему замкнутую оболочку $1 s^{2}$,

$$
G(\omega) \equiv \sum_{n} \frac{|n\rangle\langle n|}{\omega-\varepsilon_{n}+i \varepsilon_{n} 0} \rightarrow \sum_{n} \frac{|n\rangle\langle n|}{\omega-\varepsilon_{n}+i \eta_{n} 0}
$$

Здесь $\eta_{n}=\varepsilon_{n}-\varepsilon_{\mathrm{F}}$, и $\varepsilon_{\mathrm{F}}-$ энергия Ферми. Энергия Ферми выбирается так, чтобы она была выше, чем энергия электронов с замкнутой оболочки, но ниже, чем энергия валентных электронов. Переопределив вакуум и рассмотрев диаграммы для двух электронов из $L$-оболочки, мы учтем как все взаимодействия между ними, так и взаимодействие с $K$-оболочкой. Естественно, что для получения полной энергии связи бериллиеподобного иона необходимо добавить еще энергию связи самой замкнутой оболочки $1 s^{2}$, которая была рассмотрена, например, в рабо$\operatorname{Tax}[36,37]$.

Перейдем теперь к обсуждению второй задачи: обобщению формализма на произвольное количество $s$ квазивырожденных уровней, включенных в модельное подпространство $\Omega$. Для диаграмм, которые не содержат двухкратных возбуждений, соответствующее обобщение тривиально. К данным вкладам относятся все диаграммы первого порядка, одноэлектронные диаграммы второго порядка, а также двухэлектронные диаграммы собственной энергии и вакуумной поляризации. Особого внимания заслуживают диаграммы двухфотонного обмена, изображенные на рисунке. Вклад перекрестной диаграммы на рисунке $b$ обобщается без проблем. Вклад наиболее сложной лестничной диаграммы на рисунке $a$ естественно можно разделить на приводимую („red“决 и неприводимую (,irr ${ }^{6}$ ) части. Приводимая часть содержит все вклады, в которых промежуточные состояния лежат в модельном подпространстве, и переопределение вакуума соответственно на ней не сказывается. Неприводимой части соответствуют все прочие вклады. После весьма трудоемких выкладок можно получить следующие выражения для вклада лестничной диаграммы:

$$
\begin{aligned}
H_{i k}^{\mathrm{ld}, \mathrm{irr}}= & \frac{1}{2} \sum_{P}(-1)^{P} \sum_{n_{1}, n_{2}}^{E_{n}^{(0)} \neq E_{1}^{(0)} \ldots E_{s}^{(0)}} \frac{i}{2 \pi} \int_{-\infty}^{\infty} d \omega \\
& \times\left\{\frac{I_{P i_{1} P i_{2} n_{1} n_{2}}\left(\omega-\varepsilon_{P i_{1}}\right) I_{n_{1} n_{2} k_{1} k_{2}}\left(\varepsilon_{k_{1}}-\omega\right)}{\left[\omega-\varepsilon_{n_{1}}+i \eta_{n_{1}} 0\right]\left[\bar{E}_{i k}^{(0)}-\omega-\varepsilon_{n_{2}}+i \eta_{n_{2}} 0\right]}\right. \\
& \left.+\frac{I_{P i_{1} P i_{2} n_{1} n_{2}}\left(\omega-\varepsilon_{P i_{2}}\right) I_{n_{1} n_{2} k_{1} k_{2}}\left(\varepsilon_{k_{2}}-\omega\right)}{\left[\bar{E}_{i k}^{(0)}-\omega-\varepsilon_{n_{1}}+i \eta_{n_{1}} 0\right]\left[\omega-\varepsilon_{n_{2}}+i \eta_{n_{2}} 0\right]}\right\},
\end{aligned}
$$




$$
\begin{aligned}
H_{i k}^{\mathrm{ld}, \text { red }}= & -\frac{1}{2} \sum_{P}(-1)^{P} \sum_{n_{1}, n_{2}}^{E_{n}^{(0)}=E_{1}^{(0)} \ldots E_{s}^{(0)}} \frac{i}{2 \pi} \int_{-\infty}^{\infty} d \omega \\
& \times\left\{\frac{I_{P i_{1} P i_{2} n_{1} n_{2}}\left(\omega-\varepsilon_{P i_{1}}\right) I_{n_{1} n_{2} k_{1} k_{2}}\left(\varepsilon_{k_{1}}-\omega\right)}{\left[\omega-\varepsilon_{n_{1}}-i 0\right]\left[\omega+\varepsilon_{n_{2}}-\bar{E}_{i k}^{(0)}-i 0\right]}\right. \\
+ & \left.\frac{I_{P i_{1} P i_{2} n_{1} n_{2}}\left(\omega-\varepsilon_{P i_{2}}\right) I_{n_{1} n_{2} k_{1} k_{2}}\left(\varepsilon_{k_{2}}-\omega\right)}{\left[\omega+\varepsilon_{n_{1}}-\bar{E}_{i k}^{(0)}-i 0\right]\left[\omega-\varepsilon_{n_{2}}-i 0\right]}\right\},
\end{aligned}
$$

где индексы $i$ и $k$ нумеруют квазивырожденные состояния, $I_{a b c d}(\omega)=\langle a b|I(\omega)| c d\rangle, I(\omega)=e^{2} \alpha_{1}^{\mu} \alpha_{2}^{v} D_{\mu \nu}(\omega)$, $\alpha^{\mu}=(1, \boldsymbol{\alpha})$ - матрицы Дирака, $D_{\mu v}(\omega)-$ фотонный пропагатор, $P-$ оператор перестановки, $E_{n}^{(0)}=\varepsilon_{n_{1}}+\varepsilon_{n_{2}}$, и $\bar{E}_{i k}^{(0)}=\left(E_{i}^{(0)}+E_{k}^{(0)}\right) / 2$. Для простоты предполагается, что внешним волновым функциям соответствуют детерминанты Слейтера. Переход к общему случаю многодетерминантных волновых функций без труда может быть выполнен непосредственно в окончательных выражениях. Отметим, что в расчетах мы незначительно модифицируем недиагональные матричные элементы неприводимой части (3), с тем чтобы восстановить правильный предел $H_{i k}^{\text {2ph,Breit }}$ в брейтовском приближении (см. обсуждение в работе [37]). Для полноты приведем здесь также выражение для вклада перекрестной диаграммы,

$$
\begin{aligned}
& H_{i k}^{\mathrm{cr}}=\frac{1}{2} \sum_{P}(-1)^{P} \sum_{n_{1}, n_{2}} \frac{i}{2 \pi} \int_{-\infty}^{\infty} d \omega \\
& \times\left\{\frac{I_{P i_{1} n_{2} n_{1} k_{2}}\left(\omega-\varepsilon_{P i_{1}}\right) I_{n_{1} P i_{2} k_{1} n_{2}}\left(\varepsilon_{k_{1}}-\omega\right)}{\left[\omega-\varepsilon_{n_{1}}+i \eta_{n_{1}} 0\right]\left[\bar{E}_{i k}^{(0)}-\varepsilon_{P i_{1}}-\varepsilon_{k_{1}}+\omega-\varepsilon_{n_{2}}+i \eta_{n_{2}} 0\right]}\right. \\
& \left.+\frac{I_{P i_{1} n_{2} n_{1} k_{2}}\left(\varepsilon_{k_{2}}-\omega\right) I_{n_{1} P i_{2} k_{1} n_{2}}\left(\omega-\varepsilon_{P i_{2}}\right)}{\left[\bar{E}_{i k}^{(0)}-\varepsilon_{P i_{2}}-\varepsilon_{k_{2}}+\omega-\varepsilon_{n_{1}}+i \eta_{n_{1}} 0\right]\left[\omega-\varepsilon_{n_{2}}+i \eta_{n_{2}} 0\right]}\right\} .
\end{aligned}
$$

Интересно обсудить, что происходит с полным вкладом диаграмм двухфотонного обмена $H_{i k}^{2 \mathrm{ph}} \equiv H_{i k}^{\mathrm{ld}, \mathrm{irr}}+$ $+H_{i k}^{\mathrm{ld}, \mathrm{red}}+H_{i k}^{\mathrm{cr}}$ при расширении модельного подпространства. Рассмотрим модельное подпространство $\tilde{\Omega}$, отличающееся от $\Omega$ дополнительным состоянием $n$. Легко показать, что для $i, k \in \Omega$ выполняется следующее соотношение:

$$
\delta H_{i k}^{2 \mathrm{ph}}[\Omega]=\delta H_{i k}^{2 \mathrm{ph}}[\tilde{\Omega}]+\Delta_{i k},
$$

где $\delta H_{i k}^{2 \mathrm{ph}}=H_{i k}^{2 \mathrm{ph}}-H_{i k}^{2 \mathrm{ph}, \text { Breit }}-$ нетривиальная КЭД часть вклада диаграмм двухфотонного обмена (за рамками брейтовского приближения), а $\Delta_{i k}$ дается выражением

$$
\begin{aligned}
& \Delta_{i k}=\frac{1}{2} \sum_{P}(-1)^{P} \sum_{n_{1}, n_{2}}^{\varepsilon_{n_{1}}+\varepsilon_{n_{2}}=E_{n}^{(0)}} \frac{1}{\bar{E}_{i k}^{(0)}-E_{n}^{(0)}} \\
& \times\left\{D_{n_{1} n_{2}}\left(\varepsilon_{n_{1}}-\varepsilon_{P i_{1}}, \varepsilon_{k_{1}}-\varepsilon_{n_{1}}\right)\right. \\
& \left.+D_{n_{1} n_{2}}\left(\varepsilon_{n_{2}}-\varepsilon_{P i_{2}}, \varepsilon_{k_{2}}-\varepsilon_{n_{2}}\right)-2 D_{n_{1} n_{2}}(0,0)\right\},
\end{aligned}
$$

и $D_{n_{1} n_{2}}\left(\omega_{1}, \omega_{2}\right)=I_{P_{i_{1} P i_{2} n_{1} n_{2}}}\left(\omega_{1}\right) I_{n_{1} n_{2} k_{1} k_{2}}\left(\omega_{2}\right)$. Величина $\Delta_{i k}$ представляет хоть и ненулевую, но малую поправку: в наших расчетах она оказывается меньше, чем численная погрешность для соответствующих вкладов. В результате оказывается, что с высокой точностью нетривиальная КЭД часть вклада диаграмм двухфотонного обмена $\delta H_{i k}^{2 \mathrm{ph}}$ практически не изменяется при увеличении размера модельного подпространства квазивырожденных состояний, хотя сам вклад в целом меняется значительно.

Корреляционные вклады за счет обмена тремя и более фотонами учитываются в данной работе в брейтовском приближении на основе гамильтониана Дирака-Кулона-Брейта (DCB) методом наложения конфигураций (CI) в базисе орбиталей Дирака-Штурма [73,74]. Процедура, позволяющая объединить для квазивырожденных уровней КЭД расчеты в первом и втором порядках с вкладами межэлектронного взаимодействия третьего и более высоких порядков по $1 / Z$, была предложена впервые в работе [36] и изложена подробно в работе [37]. Кратко данную процедуру можно описать следующим образом. Пусть $\left\{u_{j}\right\}_{j=1}^{s}$ есть набор невозмущенных многоэлектронных функций исследуемых квазивырожденных уровней, а $\left\{E_{j}^{\mathrm{CI}}\right\}_{j=1}^{s}$ и $\left\{\Psi_{j}\right\}_{j=1}^{s}-$ соответствующие энергии и волновые функции, полученные из решения уравнения DCB. Тогда эффективный гамильтониан, описывающий корреляционные эффекты в брейтовском приближении во всех порядках по $1 / Z$, может быть записан в виде

$$
H^{\mathrm{CI}}=P^{-1 / 2} K P^{-1 / 2},
$$

где матрицы $K$ и $P$ определены следующим образом:

$$
\begin{gathered}
K_{i k}=\sum_{j=1}^{s} E_{j}^{\mathrm{CI}}\left\langle u_{i} \mid \Psi_{j}\right\rangle\left\langle\Psi_{j} \mid u_{k}\right\rangle, \\
P_{i k}=\sum_{j=1}^{s}\left\langle u_{i} \mid \Psi_{j}\right\rangle\left\langle\Psi_{j} \mid u_{k}\right\rangle .
\end{gathered}
$$

Чтобы получить вклад высших порядков, необходимо вычесть из матрицы $H^{\mathrm{CI}}$ вклады нулевого, первого и второго порядков по $1 / Z$, рассчитанные по теории возмущений.

Наконец, помимо упомянутых выше вкладов, при расчете энергий связи бериллиеподобных ионов мы принимаем во внимание также эффекты отдачи и поляризации 
Таблица 1. Энергия связи (с обратным знаком) основного состояния в бериллиеподобном молибдене (в eV). Сравнение различных схем расчета $(1 \times 1,2 \times 2$ и $3 \times 3)$ и подходов (A, B, C, и D)

\begin{tabular}{c|c|c|c|c|c}
\hline$\Omega$ & $V_{\text {eff }}$ & $\mathrm{A}$ & $\mathrm{B}$ & $\mathrm{C}$ & $\mathrm{D}$ \\
\hline \multirow{3}{*}{$1 \times 1$} & $V_{\text {nucl }}$ & 59689.239 & 59644.885 & 59646.346 & - \\
& $\mathrm{CH}$ & 59689.266 & 59646.913 & 59646.317 & 59646.623 \\
& $\mathrm{LDF}$ & 59689.257 & 59646.622 & 59646.319 & 59646.536 \\
\hline \multirow{3}{*}{$2 \times 2$} & $V_{\text {nucl }}$ & 59689.239 & 59644.991 & 59646.435 & 59646.535 \\
& $\mathrm{CH}$ & 59689.266 & 59647.009 & 59646.404 & 59646.469 \\
& $\mathrm{LDF}$ & 59689.257 & 59646.718 & 59646.407 & 59646.457 \\
\hline \multirow{4}{*}{$3 \times 3$} & $V_{\text {nucl }}$ & 59689.239 & 59645.043 & 59646.475 & 59646.455 \\
& $\mathrm{CH}$ & 59689.266 & 59647.057 & 59646.445 & 59646.456 \\
& $\mathrm{LDF}$ & 59689.257 & 59646.765 & 59646.448 & 59646.452
\end{tabular}

ядра. Для случая гелиеподобных ионов процедура учета эффекта отдачи ядра была подробно изложена в работе [75]. В данной работе мы обобщили соответствующую процедуру на случай четырехэлектронных состояний. Эффект поляризации ядра рассмотрен в соответствии с работой [12].

\section{3. Результаты и обсуждение}

Перейдем к обсуждению полученных результатов. Все расчеты выполнены как для кулоновского потенциала ядра $V_{\text {nucl }}$ в качестве потенциала нулевого приближения в уравнении (1), так и в рамках расширенной версии картины Фарри. Во втором случае нами были использованы два различных экранирующих потенциала: потенциал Хартри $(\mathrm{CH})$ [65] и локальный потенциал Дирака-Фока (LDF) [76]. Для описания распределения заряда ядра использована модель Ферми с параметром толщины оболочки равным $t=2.3 \mathrm{fm}$. Значение среднеквадратичного радиуса для ядра молибдена взято из табуляции [77] и равно $\left\langle r^{2}\right\rangle^{1 / 2}=4.4091(18) \mathrm{fm}$, что соответствует изотопу с массовым числом $A=98$. Отношение массы ядра к массе электрона $M / m=178428$ взято из компиляции Ame2012 [78]. Использованы значения фундаментальных констант, рекомендованные CODATA 2014 [79]: $\alpha^{-1}=137.035999139(31)$ и $m c^{2}=0.5109989461(31) \mathrm{MeV}$. Численный расчет одноэлектронных волновых функций-решений уравнения (1), необходимых для представления функции Грина (2), выполнен, используя метод дуального кинетического баланса [80] с базисными функциям, составленными из $B$-сплайнов $[81,82]$. Интегрирование по $\omega$ в выражениях (3)-(5) и аналогичных осуществлено, используя стандартный поворот Вика. В одноэлектронных и двухэлектронных вкладах вакуумной поляризации радиальная часть функции Грина найдена численно путем решения системы соответствующих дифференциальных уравнений.
В табл. 1 представлены результаты расчетов энергии связи основного состояния в бериллиеподобном молибдене (с обратным знаком). Применены три альтернативных схемы расчета: сперва основное состояние рассмотрено как одиночный уровень, затем использована теория возмущений для квазивырожденных уровней с двумерным и трехмерным модельным подпространством $\Omega$. Тип теории возмущений указан в первом столбце, где приведен размер $s \times s$ матрицы эффективного гамильтониана $H$. Столбцы, помеченные буквами $\mathrm{A}, \mathrm{B}, \mathrm{C}$ и $\mathrm{D}$, демонстрируют эволюцию результатов по мере того, как в расчеты добавляются различные вклады. В столбце А показаны результаты, полученные в брейтовском приближении методом CI, т.е. приведены собственные числа матрицы $H^{\mathrm{CI}}$ из уравнения (8). Из табл. 1 можно видеть, что для конкретного потенциала результаты в этом столбце не зависят от типа теории возмущений. Это неудивительно, поскольку в методе CI корреляционные эффекты учитываются во всех порядках по $1 / Z$. Если в выражении (8) ограничиться конечным порядком теории возмущений, например, рассмотреть только вклады нулевого, первого и второго порядков по $1 / Z$, то результаты, естественно, будут различаться. С другой стороны, для конкретного модельного подпространства $\Omega$ результаты в столбце А изменяются слегка при переходе от одного потенциала к другому. Данные изменения можно объяснить зависимостью проекторов на состояния положительного спектра в гамильтониане DCB от выбора начального приближения (см. соответствующее обсуждение, например, в работах $[37,83])$.

Значения в столбце В получены путем включения в расчеты КЭД поправок первого порядка, а именно были добавлены вклады одноэлектронных диаграмм собственной энергии и вакуумной поляризации и поправка на частотную зависимость к вкладу диаграммы однофотонного обмена. Кроме того, в столбце В учтены вклады эффектов отдачи и поляризации ядра. Как отмечалось выше, в предыдущих релятивистских расчетах энергий бериллиеподобных ионов рассмотрение КЭД поправок было ограничено именно этим уровнем точности $[41,42,46]$. Результаты для разных потенциалов в столбце В демонстрируют значительный разброс, который может быть уменьшен только за счет добавления КЭД поправок второго порядка. Это показано в столбце С, где в расчет включены вклады двухэлектронных диаграмм собственной энергии и вакуумной поляризации, нетривиальная КЭД часть вклада диаграмм двухфотонного обмена (за рамками брейтовского приближения), а также одноэлектронные двухпетлевые поправки. Для кулоновского потенциала двухпетлевые поправки взяты из работы [12], а для потенциалов $\mathrm{CH}$ и LDF с целью учета эффектов экранирования кулоновские значения были масштабированы, используя КЭД поправки первого порядка для состояний $n s$, где $n-$ главное квантовое число. Из табл. 1 
можно увидеть, что различия между расчетами, выполненными для кулоновского и двух экранирующих потенциалов, действительно уменьшаются при переходе от столбца В к столбцу С. Кроме того, можно заключить, что значения, полученные для потенциалов СH и LDF в столбце $\mathrm{C}$, близки друг к другу, но слегка сдвинуты по сравнению с соответствующими кулоновскими значениями. Это является результатом перестраивания рядов теории возмущений в расширенной версии картины Фарри, что дает возможность частично учесть КЭД вклады более высокого порядка.

Важная особенность КЭД теории возмущений для квазивырожденных уровней заключается в том, что калибровочно инвариантными являются собственные числа $\left\{E_{j}\right\}_{j=1}^{s}$ матрицы $H$, рассчитанной во всех порядках по $\alpha$ и $1 / Z$. Отдельные вклады в эффективный гамильтониан определенного порядка по $1 / Z$ могут варьироваться от калибровки к калибровке. Это связано с тем, что собственные числа матрицы нелинейным образом связаны с ее матричными элементами, и, как результат, различные порядки теории возмущений для диагональных и недиагональных матричных элементов перемешиваются при диагонализации. Например, для двумерного подпространства $\Omega$ имеем

$$
E_{1,2}=\frac{H_{11}+H_{22} \pm \sqrt{\left(H_{11}-H_{22}\right)^{2}+4 H_{12} H_{21}}}{2} .
$$

Следует подчеркнуть, что матрица $H$ в целом определена неоднозначно, с точностью до унитарного преобразования. В данной работе мы нацелены на объединение КЭД расчетов с корреляционными вкладами высших порядков в брейтовском приближении. Гамильтониан DCB естественным образом связан с кулоновской калибровкой [84], поэтому в КЭД расчетах для виртуального фотона, отвечающего за межэлектронное взаимодействие, мы используем именно эту калибровку (данные, представленные в табл. 1 в столбцах В и С, получены в кулоновской калибровке). Однако для сравнения мы также проводим расчеты в фейнмановской калибровке. В частности, при использовании теории возмущений для одиночного уровня отдельные вклады, рассчитанные в разных калибровках, хорошо согласуются друг с другом. Кроме того, в силу калибровочной инвариантности собственных чисел матрицы $H$ аналогичным свойством обладает и след матрицы: $\operatorname{Tr} H=\sum_{j} H_{j j}=\sum_{j} E_{j}$. При этом, поскольку след линейно зависит от элементов матрицы $H$, калибровочная инвариантность должна иметь место для всех порядков теории возмущений в отдельности. Для первого порядка по $1 / Z$ данный факт тривиален: диагональные матричные элементы для диаграммы однофотонного обмена не зависят от калибровки. Поэтому представляет интерес проследить за выполнением данного утверждения во втором порядке по $1 / Z$ для диаграмм двухфотонного обмена. Это послужит косвенной проверкой корректности проведенных расчетов.
Таблица 2. Разница кулоновской и фейнмановской калибровок для вклада диаграмм двухфотонного обмена в диагональные матричные элементы матрицы $H$ (в $\mathrm{meV})$. Состояния $(2 s 2 s)_{0},\left(2 p_{1 / 2} 2 p_{1 / 2}\right)_{0}$ и $\left(2 p_{3 / 2} 2 p_{3 / 2}\right)_{0}$ обозначают соответствующие диагональные элементы

\begin{tabular}{c|c|c|c|c|c}
\hline$\Omega$ & $V_{\text {eff }}$ & $(2 s 2 s)_{0}$ & $\left(2 p_{1 / 2} 2 p_{1 / 2}\right)_{0}$ & $\left(2 p_{3 / 2} 2 p_{3 / 2}\right)_{0}$ & Сумма \\
\hline \multirow{3}{*}{$2 \times 2$} & $V_{\text {nucl }}$ & 0.00 & 0.00 & - & 0.00 \\
& CH & -0.13 & 0.13 & - & 0.00 \\
& LDF & -0.16 & 0.16 & - & 0.00 \\
\hline \multirow{3}{*}{$3 \times 3$} & $V_{\text {nucl }}$ & -0.53 & -0.04 & $0.56(3)$ & $0.00(3)$ \\
& CH & -0.88 & 0.10 & $0.77(2)$ & $0.00(2)$ \\
& LDF & -0.97 & 0.13 & $0.83(2)$ & $0.00(2)$
\end{tabular}

Действительно, оказывается, что хотя отдельные диагональные матричные элементы изменяются при смене калибровки виртуальных фотонов, их сумма с высокой степенью точности сохраняется. Данный результат продемонстрирован в табл. 2, где для матриц размером $2 \times 2$ и $3 \times 3$ показана разница между результатами расчетов в кулоновской и фейнмановской калибровках. Данные для матрицы размером $1 \times 1$ опущены в силу их калибровочной инвариантности. Заканчивая обсуждения данного вопроса, отметим, что если переразложить собственные числа матрицы в ряд по $\alpha$ и $1 / Z$, то для отдельных членов ряда естественно также будет выполняться калибровочная инвариантность. Так, например, если в формуле (11) ограничиться вкладами первого и второго порядков по $1 / Z$, рассмотрев только диаграммы одно- и двухфотонного обменов, то можно получить

$$
\begin{gathered}
E_{1} \approx E_{1}^{(0)}+H_{11}^{1 \mathrm{ph}}+\left(H_{11}^{2 \mathrm{ph}}+\delta E^{(2)}\right), \\
E_{2} \approx E_{2}^{(0)}+H_{22}^{1 \mathrm{ph}}+\left(H_{22}^{2 \mathrm{ph}}-\delta E^{(2)}\right), \\
\delta E^{(2)}=\frac{H_{12}^{1 \mathrm{ph}} H_{21}^{1 \mathrm{ph}}}{E_{1}^{(0)}-E_{2}^{(0)}} .
\end{gathered}
$$

Недиагональный матричный элемент $H_{12}^{1 \mathrm{ph}}$ для диаграммы однофотонного обмена не калибровочно инвариантен. Отличие величины $\delta E^{(2)}$, рассчитанной в кулоновской и фейнмановской калибровках, составляет 0.00 , 0.16 и 0.13 (в единицах $\mathrm{meV}$ ) для кулоновского, $\mathrm{CH}$ и $\mathrm{LDF}$ потенциалов соответственно. С учетом данных, представленных в табл. 2, можно видеть, что вклады второго порядка по $1 / Z$ в круглых скобках в уравнениях (12) и (13) действительно не зависят от выбора калибровки. Справедливость аналогичного утверждения можно показать и для матрицы размером $3 \times 3$, однако это потребует более сложных выкладок.

Вернемся к обсуждению табл. 1. Из столбца С можно видеть, что при переходе от $1 \times 1$ к $2 \times 2$ энергия связи основного состояния заметно опускается. Однако величина этого изменения (порядка $-88 \mathrm{meV}$ ) практически 
не зависит от выбора начального приближения в уравнении (1). При расширении модельного подпространства $\Omega$ за счет добавления конфигурации $\left(2 p_{3 / 2} 2 p_{3 / 2}\right)_{0}$ энергия претерпевает дополнительный сдвиг порядка $-41 \mathrm{meV}$. Данные изменения объясняются учетом перемешивания состояний в модельном подпространстве. По сравнению с теорией возмущений для одиночного уровня при построении матрицы эффективного гамильтониана и расчете ее собственных чисел мы эффективно суммируем важные члены более высокого порядка, описывающие взаимодействие внутри $\Omega$. Тот факт, что эффект добавления конфигурации $\left(2 p_{3 / 2} 2 p_{3 / 2}\right)_{0}$ сравним с эффектом включения конфигурации $\left(2 p_{1 / 2} 2 p_{1 / 2}\right)_{0}$ означает, что обе они одинаково важны для надлежащего описания основного состояния в бериллиеподобном молибдене [38]. Подчеркнем, что именно применение КЭД теории возмущений для квазивырожденных уровней позволяет нам правильно учесть взаимное влияние корреляционных и КЭД эффектов на основное состояние бериллиеподобного молибдена.

Погрешность расчетов, представленных в табл. 1 в столбце C, определяется, в частности, неучтенными экранированными КЭД вкладами второго порядка по $1 / Z$. В настоящее время данные поправки недоступны для строгих расчетов методами квантовой электродинамики для связанных состояний. Однако их можно оценить приближенно, воспользовавшись модельным оператором лэмбовского сдвига (QEDMOD), который был предложен в работах $[85,86]$. Оператор QEDMOD положительно зарекомендовал себя в расчетах различных атомных систем [31,48,83,87-90]. С целью оценки экранированных КЭД поправок второго порядка по $1 / Z$ мы добавили оператор QEDMOD в уравнение Дирака (1) и с помощью соответствующего одноэлектронного базиса в брейтовском приближении рассчитали вклад диаграмм двухфотонного обмена. Искомая поправка была получена путем вычитания вклада $H_{i k}^{2 \mathrm{ph}, \text { Breit }}$, рассчитанного без оператора QEDMOD. Энергии связи с учетом последней поправки показаны в столбце D. Можно видеть, что результаты невырожденной теории возмущений для потенциалов CH и LDF сдвигаются в сторону собственных чисел соответствующих матриц $H$ и даже „перескакивают“ их, при этом различие между значениями для двух экранирующих потенциалов оказывается большим. Это означает, что в рамках теории возмущений для одиночного уровня КЭД эффекты высших порядков вносят значительный вклад, которым нельзя пренебрегать. Напротив, различие между результатами для подпространств размером $2 \times 2$ и $3 \times 3$ становится меньше при переходе от столбца С к столбцу D. Более того, кулоновское значение для матрицы максимального размера практически сравнивается с соответствующими значениями, полученными для экранирующих потенциалов. Окончательно на основе всех результатов, представленных в табл. 1, мы приходим к выводу, что трехмерное модельное подпространство, включающее конфигурации
Таблица 3. Энергия связи основного состояния в бериллиеподобном молибдене (в еV)

\begin{tabular}{l|c|l}
\hline \multicolumn{1}{c|}{$E$} & Год & \multicolumn{1}{|c}{ Ссылка } \\
\hline$-59646.452(16)$ & 2021 & Данная работа \\
$-59646.51(84)$ & 2019 & Kaygorodov et al. $[83]$ \\
$-59646.283(54)^{\dagger}$ & 2014 & Malyshev et al. $[60]$ \\
-59647.22 & 2005 & Gu [45] \\
-59646.896 & 1997 & Chen \& Cheng [42]
\end{tabular}

Примечание. † Энергия пересчитана, используя рекомендованные CODATA 2014 значения фундаментальных констант [79].

$(2 s 2 s)_{0}, \quad\left(2 p_{1 / 2} 2 p_{1 / 2}\right)_{0}$ и $\left(2 p_{3 / 2} 2 p_{3 / 2}\right)_{0}$, является достаточным для корректного описания корреляционных и КЭД эффектов для энергии связи основного состояния в бериллиеподобном молибдене на рассматриваемом уровне точности.

Значение, представленное в табл. 1 для потенциала LDF и максимального размера $3 \times 3$ модельного подпространства $\Omega$, выбрано нами в качестве окончательного результата. Теоретическая погрешность, указанная в табл. 3, получена путем квадратичного сложения нескольких вкладов. В первую очередь помимо численных погрешностей мы принимаем во внимание погрешности, связанные с эффектом конечного размера ядра и одноэлектронными двухпетлевыми поправками [12]. Погрешность, связанная с конечным размером ядра, оценена консервативно посредством варьирования среднеквадратичного радиуса в указанных в работе [77] пределах, а также путем сравнения с результатами расчетов, выполненных для модели распределения заряда, соответствующей однородно заряженному шару. Во-вторых, несколькими способами оценены неучтенные КЭД вклады высших порядков. Квантово-электродинамические поправки к вкладам электрон-электронных корреляций третьего и более высоких порядков оценены согласно процедуре, описанной в работе [37]. Эффекты экранирования межэлектронным взаимодействием для двухпетлевых поправок оценены при помощи умножения соответствующего вклада для состояния $1 s$ на консервативный множитель $2 / Z$. Наконец, поскольку наши расчеты экранированных КЭД эффектов второго порядка по $1 / Z$, представленные в табл. 1 в столбце D, носят приближенный характер, мы включили соответствующую поправку с 100\% погрешностью.

В табл. 3 мы сравниваем полученную энергию связи основного состояния бериллиеподобного молибдена с результатами предыдущих релятивистских расчетов. В первую очередь следует отметить, что новое значение отличается от представленного нами ранее в работе 2014 г. [60], где была применена теория возмущений для одиночного уровня. Мы вынуждены признать, что КЭД эффекты высших порядков были нами сильно недооценены тогда. Мы рассматриваем использованную сейчас процедуру для оценки теоретической погреш- 
ности как более надежную по сравнению с той, что применялась ранее в работе [60], поскольку она основана на всестороннем анализе результатов, полученных в рамках альтернативных схем расчета (разные типы теории возмущений) для различных потенциалов нулевого приближения, а также на расчете КЭД эффектов высших порядков посредством оператора QEDMOD. Мы обращаем внимание, что текущее значение, представленное в табл. 1 в столбце С для невырожденной теории возмущений $(1 \times 1)$ и для потенциала LDF, получено в рамках подхода, который согласуется с использованным в работе [60]. Данное значение смещено относительно предыдущего в пределах обозначенной погрешности. Данный сдвиг объясняется изменениями в подходе к расчету в брейтовском приближении вкладов межэлектронного взаимодействия высших порядков, а также скорректированными данными для одноэлектронных двухпетлевых поправок. В работе [83] энергии связи бериллиеподобных ионов были рассчитаны методом CI + QEDMOD, при этом погрешности были оценены весьма консервативно. Сравнение с результатом, полученным в данной работе, показывает, что использованный в работе [83] подход имеет точность по крайней мере на один порядок величины выше.

\section{4. Заключение}

Проведены расчеты энергии связи основного состояния бериллиеподобного молибдена с привлечением самых передовых КЭД методов из доступных на сегодня. Вклады всех фейнмановских диаграмм до второго порядка рассмотрены в рамках картины Фарри. Корреляционные эффекты высших порядков учтены в брейтовском приближении. Впервые основное состояние бериллиеподобных ионов исследовано при помощи КЭД теории возмущений для квазивырожденных уровней с трехмерным модельным подпространством, включающим состояния $1 s^{2}(2 s 2 s)_{0}, 1 s^{2}\left(2 p_{1 / 2} 2 p_{1 / 2}\right)_{0}$ и $1 s^{2}\left(2 p_{3 / 2} 2 p_{3 / 2}\right)_{0}$. Выявлена существенная взаимосвязь между корреляционными и КЭД эффектами. В результате получены наиболее точные теоретические предсказания для энергии связи основного состояния. В будущем мы планируем применить разработанный подход к анализу энергий возбужденных состояний с полным угловым моментом равным $J=1$ и расчету соответствующих переходов $2 s 2{ }^{1} S_{0}-2 s 2 p^{1} P_{1}$ и $2 s 2 s{ }^{1} S_{0}-2 s 2 p^{3} P_{1}$ в бериллиеподобном молибдене.

\section{Финансирование работы}

Исследование выполнено за счет гранта Президента Российской Федерации (проект № МК-1459.2020.2), а также при финансовой поддержке РФФИ и Госкорпорации „Росатом“ в рамках научного проекта № 20-2100098. Работа также поддержана грантом РФФИ № 1902-00974. А.В. Малышев, М.Ю. Кайгородов, В.М. Шаба- ев, Д.А. Глазов благодарят за поддержку Фонд развития теоретической физики и математики „БАЗИС“.

\section{Конфликт интересов}

Авторы заявляют, что у них нет конфликта интересов.

\section{Список литературы}

[1] Beyer H.F., Shevelko V.P. Introduction to the Physics of Highly Charged Ions. Institute of Physics Publishing, Bristol and Philadelphia, 2003.

[2] Shabaev V.M., Bondarev A.I., Glazov D.A., Kaygorodov M.Y., Kozhedub Y.S., Maltsev I.A., Malyshev A.V., Popov R.V., Tupitsyn I.I., Zubova N.A. // Hyp. Interact. 2018. V. 239. P. 60.

[3] Indelicato P. // J. Phys. B: At. Mol. Opt. Phys. 2019. V. 52. P. 232001.

[4] Korobov V., Yelkhovsky A. // Phys. Rev. Lett. 2001. V. 87. P. 193003.

[5] Pachucki K., Patkóš V., Yerokhin V.A. // Phys. Rev. A. 2017. V. 95. P. 062510.

[6] Stöhlker Th., Mokler P.H., Bosch F., Dunford R.W., Franzke F., Klepper O., Kozhuharov C., Ludziejewski T., Nolden F., Reich H., Rymuza P., Stachura Z., Steck M., Swiat P., Warczak A. // Phys. Rev. Lett. 2000. V. 85. P. 3109.

[7] Gumberidze A., Stöhlker Th., Banaś D., Beckert K., Beller P., Beyer H.F., Bosch F., Hagmann S., Kozhuharov C., Liesen D., Nolden F., Ma X., Mokler P.H., Steck M., Sierpowski D., Tashenov S. // Phys. Rev. Lett. 2005. V. 94. P. 223001.

[8] Schweppe J., Belkacem A., Blumenfeld L., Claytor N., Feinberg B., Gould H., Kostroun V.E., Levy L., Misawa S., Mowat J.R., Prior M.H. // Phys. Rev. Lett. 1991. V. 66. P. 1434.

[9] Brandau C., Kozhuharov C., Müller A., Shi W., Schippers S., Bartsch T., Böhm S., Böhme C., Hoffknecht A., Knopp H., Grün N., Scheid W., Steih T., Bosch F., Franzke B., Mokler P.H., Nolden F., Steck M., Stöhlker T., Stachura Z. // Phys. Rev. Lett. 2003. V. 91. P. 073202.

[10] Beiersdorfer P., Chen H., Thorn D.B., Träbert E. // Phys. Rev. Lett. 2005. V. 95. P. 233003.

[11] Johnson W.R., Soff G. // At. Data Nucl. Data Tables. 1985. V. 33. P. 405.

[12] Yerokhin V.A., Shabaev V.M. // J. Phys. Chem. Ref. Data. 2015. V. 44. P. 033103.

[13] Johnson W.R., Blundell S.A., Sapirstein J. // Phys. Rev. A. 1988. V. 37. P. 2764.

[14] Indelicato P., Desclaux J.P. // Phys. Rev. A. 1990. V. 42. P. 5139.

[15] Yerokhin V.A., Artemyev A.N., Shabaev V.M. // Phys. Rev. A. 2007. V. 75. P. 062501.

[16] Yerokhin V.A., Artemyev A.N., Beier T., Plunien G., Shabaev V.M., Soff G. // Phys. Rev. A. 1999. V. 60. P. 3522.

[17] Artemyev A.N., Beier T., Plunien G., Shabaev V.M., Soff G., Yerokhin V.A. // Phys. Rev. A. 1999. V. 60. P. 45.

[18] Yerokhin V.A., Artemyev A.N., Shabaev V.M., Sysak M.M., Zherebtsov O.M., Soff G. // Phys. Rev. A. 2001. V. 64. P. 032109.

[19] Kozhedub Y.S., Andreev O.V., Shabaev V.M., Tupitsyn I.I., Brandau C., Kozhuharov C., Plunien G., Stöhlker T. // Phys. Rev. A. 2008. V. 77. P. 032501. 
[20] Sapirstein J., Cheng K.T. // Phys. Rev. A. 2011. V. 83. P. 012504.

[21] Yerokhin V.A., Puchalski M., Pachucki K. // Phys. Rev. A. 2020. V. 102. P. 042816.

[22] Yerokhin V.A., Surzhykov A. // J. Phys. Chem. Ref. Data. 2018. V. 47. P. 023105.

[23] Зайцев В.А., Мальцев И.А., Тупищын И.И., Шабаев В.М., Иванов В.Ю. // Опт. спектр. 2020. V. 128. Р. 318.

[24] Yerokhin V.A., Artemyev A.N., Shabaev V.M. // Phys. Lett. A. 1997. V. 234. P. 361.

[25] Beiersdorfer P., Bitter M., von Goeler S., Hill K.W. // Phys. Rev. A. 1989. V. 40. P. 150.

[26] Kukla K.W., Livingston A.E., Suleiman J., Berry H.G., Dunford R.W., Gemmell D.S., Kanter E.P., Cheng S., Curtis L.J. // Phys. Rev. A. 1995. V. 51. P. 1905.

[27] Chantler C.T., Kinnane M.N., Gillaspy J.D., Hudson L.T., Payne A.T., Smale L.F., Henins A., Pomeroy J.M., Tan J.N., Kimpton J.A., Takacs E., Makonyi K. // Phys. Rev. Lett. 2012. V. 109. P. 153001.

[28] Kubiček K., Mokler P.H., Mäckel V., Ullrich J., Crespo López-Urrutia J.R. // Phys. Rev. A. 2014. V. 90. P. 032508.

[29] Epp S.W., Steinbrügge R., Bernitt S., Rudolph J.K., Beilmann C., Bekker H., Müller A., Versolato O.O., Wille H.C., Yavaş H., Ullich J., Crespo López-Urrutia J.R. // Phys. Rev. A. 2015. V. 92. P. 020502(R).

[30] Beiersdorfer P., Brown G.V. // Phys. Rev. A. 2015. V. 91. P. 032514.

[31] Machado J., Szabo C.I., Santos J.P., Amaro P., Guerra M., Gumberidze A., Guojie Bian, Isac J.M., Indelicato P. // Phys. Rev. A. 2018. V. 97. P. 032517.

[32] Drake G.W. // Can. J. Phys. 1988. V. 66. P. 586.

[33] Johnson W.R., Sapirstein J. // Phys. Rev. A. 1992. V. 46. P. R2197.

[34] Plante D.R., Johnson W.R., Sapirstein J. // Phys. Rev. A. 1994. V. 49. P. 3519.

[35] Artemyev A.N., Shabaev V.M., Yerokhin V.A., Plunien G., Soff G. // Phys. Rev. A. 2005. V. 71. P. 062104.

[36] Malyshev A.V., Kozhedub Y.S., Glazov D.A., Tupitsyn I.I., Shabaev V.M. // Phys. Rev. A. 2019. V. 99. P. 010501(R).

[37] Kozhedub Y.S., Malyshev A.V., Glazov D.A., Shabaev V.M., Tupitsyn I.I. // Phys. Rev. A. 2019. V. 100. P. 062506.

[38] Браун М.А., Гурчумелия А.Д., Сафронова У.И. Релятивистская теория атома. М.: Наука, 1984.

[39] Lindroth E., Hvarfner J. // Phys. Rev. A. 1992. V. 45. P. 2771.

[40] Zhu X.W., Chung K.T. // Phys. Rev. A. 1994. V. 50. P. 3818.

[41] Safronova M.S., Johnson W.R., Safronova U.I. // Phys. Rev. A. 1996. V. 53. P. 4036.

[42] Chen M.H., Cheng K.T. // Phys. Rev. A. 1997. V. 55. P. 166.

[43] Safronova U.I. // Mol. Phys. 2000. V. 98. P. 1213.

[44] Majumder S., Das B.P. // Phys. Rev. A. 2000. V. 62. P. 042508.

[45] Gu M.F. // At. Data Nucl. Data Tables. 2005. V. 89. P. 267.

[46] Cheng K.T., Chen M.H., Johnson W.R. // Phys. Rev. A. 2008. V. 77. P. 052504

[47] Sampaio J.M., Parente F., Nazé C., Godefroid M., Indelicato P., Marques J.P. // Phys. Scr. 2013. V. T156. P. 014015.

[48] Yerokhin V.A., Surzhykov A., Fritzsche S. // Phys. Rev. A. 2014. V. 90. P. 022509.

[49] Edlén B. // Phys. Scr. 1983. V. 28. P. 51.

[50] Möller G., Träbert E., Lodwig V., Wagner C., Heckmann P.H., Blanke J.H., Livingston A.E., Mokler P.H. // Z. Phys. D. 1989. V. 11. P. 333.
[51] Träbert E., Möller G., Heckmann P.H., Livingston A.E. // Phys. Scr. 1990. V. 41. P. 860.

[52] Möller G., Träbert E., Heckmann P.H., Mokler P.H., Livingston A.E. // Z. Phys. D. 1991. V. 18. P. 223.

[53] Büttner R., Kraus B., Schartner K.H., Folkmann F., Mokler P.H., Möller G. // Z. Phys. D. 1992. V. 22. P. 693.

[54] Beiersdorfer P., Knapp D., Marrs R.E., Elliott S.R., Chen M.H. // Phys. Rev. Lett. 1993. V. 71. P. 3939.

[55] Beiersdorfer P., Osterheld A., Elliott S.R., Chen M.H., Knapp D., Reed K. // Phys. Rev. A. 1995. V. 52. P. 2693.

[56] Träbert E., Beiersdorfer P., Lepson J.K., Chen H. // Phys. Rev. A. 2003. V. 68. P. 042501.

[57] Draganić I., Crespo López-Urrutia J.R., DuBois R., Fritzsche S., Shabaev V.M., Soria Orts R., Tupitsyn I.I., Zou Y., Ullrich J. // Phys. Rev. Lett. 2003. V. 91. P. 183001.

[58] Feili D., Zimmermann B., Neacsu C., Bosselmann P., Schartner K.H., Folkmann F., Livingston A.E., Träbert E., Mokler P.H. // Phys. Scr. 2005. V. 71. P. 48.

[59] Bernhardt D., Brandau C., Harman Z., Kozhuharov C., Böhm S., Bosch F., Fritzsche S., Jacobi J., Kieslich S., Knopp H., Nolden F., Shi W., Stachura Z., Steck M., Stöhlker Th., Schippers S., Müller A. // J. Phys. B: At. Mol. Opt. Phys. 2015. V. 48. P. 144008.

[60] Malyshev A.V., Volotka A.V., Glazov D.A., Tupitsyn I.I., Shabaev V.M., Plunien G. // Phys. Rev. A. 2014. V. 90. P. 062517.

[61] Malyshev A.V., Volotka A.V., Glazov D.A., Tupitsyn I.I., Shabaev V.M., Plunien G. // Phys. Rev. A. 2015. V. 92. P. 012514.

[62] Kozhedub Y.S., Volotka A.V., Artemyev A.N., Glazov D.A., Plunien G., Shabaev V.M., Tupitsyn I.I., Stöhlker Th. // Phys. Rev. A. 2010. V. 81. P. 042513.

[63] Denne B., Magyar G., Jacquinot J. // Phys. Rev. A. 1989. V. 40. P. 3702.

[64] Furry W.H. // Phys. Rev. 1951. V. 81. P. 115.

[65] Sapirstein J., Cheng K.T. // Phys. Rev. A. 2001. V. 64. P. 022502.

[66] Sapirstein J., Cheng K.T. // Phys. Rev. A. 2003. V. 68. P. 042111.

[67] Sapirstein J., Cheng K.T. // Phys. Rev. A. 2006. V. 74. P. 042513.

[68] Artemyev A.N., Shabaev V.M., Tupitsyn I.I., Plunien G., Yerokhin V.A. // Phys. Rev. Lett. 2007. V. 98. P. 173004.

[69] Malyshev A.V., Glazov D.A., Volotka A.V., Tupitsyn I.I., Shabaev V.M., Plunien G., Stöhlker Th. // Phys. Rev. A. 2017. V. 96. P. 022512.

[70] Shabaev V.M. // Phys. Rep. 2002. V. 356. P. 119.

[71] Le Bigot É.O., Indelicato P., Shabaev V.M. // Phys. Rev. A. 2001. V. 63. P. 040501(R).

[72] Artemyev A.N., Beier T., Plunien G., Shabaev V.M., Soff G., Yerokhin V.A. // Phys. Rev. A. 2000. V. 62. P. 022116.

[73] Братиев В.Ф., Дейнека Г.Б., Тупицын И.И. // Изв. Акад. наук СССР: сер. Физ. 1977. V. 41. Р. 2655.

[74] Tupitsyn I.I., Shabaev V.M., Crespo López-Urrutia J.R., Draganić I., Soria Orts R., Ullich J. // Phys. Rev. A. 2003. V. 68. P. 022511.

[75] Malyshev A.V., Popov R.V., Shabaev V.M., Zubova N.A. // J. Phys. B: At. Mol. Opt. Phys. 2018. V. 51. P. 085001.

[76] Shabaev V.M., Tupitsyn I.I., Pachucki K., Plunien G., Yerokhin V.A. // Phys. Rev. A. 2005. V. 72. P. 062105.

[77] Angeli I., Marinova K.P. // At. Data Nucl. Data Tables. 2013. V. 99. P. 69 
[78] Wang M., Audi G., Wapstra A.H., Kondev F.G., MacCormick M., Xu X., Pfeiffer B. // Chin. Phys. C. 2012. V. 36. P. 1603.

[79] Mohr P.J., Newell D.B., Taylor B.N. // Rev. Mod. Phys. 2016. V. 88. P. 035009.

[80] Shabaev V.M., Tupitsyn I.I., Yerokhin V.A., Plunien G., Soff G. // Phys. Rev. Lett. 2004. V. 93. P. 130405.

[81] Johnson W.R., Blundell S.A., Sapirstein J. // Phys. Rev. A. 1988. V. 37. P. 307.

[82] Sapirstein J., Johnson W.R. // J. Phys. B: At. Mol. Opt. Phys. 1996. V. 29. P. 5213.

[83] Kaygorodov M.Y., Kozhedub Y.S., Tupitsyn I.I., Malyshev A.V., Glazov D.A., Plunien G., Shabaev V.M. // Phys. Rev. A. 2019. V. 99. P. 032505.

[84] Shabaev V.M. // J. Phys. B: At. Mol. Opt. Phys. 1993. V. 26. P. 4703.

[85] Shabaev V.M., Tupitsyn I.I., Yerokhin V.A. // Phys. Rev. A. 2013. V. 88. P. 012513.

[86] Shabaev V.M., Tupitsyn I.I., Yerokhin V.A. // Comp. Phys. Commun. 2018. V. 223. P. 69

[87] Tupitsyn I.I., Kozlov M.G., Safronova M.S., Shabaev V.M., Dzuba V.A. // Phys. Rev. Lett. 2016. V. 117. P. 253001.

[88] Pašteka L.F., Eliav E., Borschevsky A., Kaldor U., Schwerdtfeger P. // Phys. Rev. Lett. 2017. V. 118. P. 023002.

[89] Zaytsev V.A., Maltsev I.A., Tupitsyn I.I., Shabaev V.M. // Phys. Rev. A. 2019. V. 100. P. 052504.

[90] Shabaev V.M., Tupitsyn I.I., Kaygorodov M.Y., Kozhedub Y.S., Malyshev A.V., Mironova D.V. // Phys. Rev. A. 2020. V. 101. P. 052502 . 